\title{
Excess Volume in Grain Boundary Segregation
}

\author{
Pavel Lejček, ${ }^{1 *}$ Lei Zheng ${ }^{2}$ and Ye Meng ${ }^{2}$ \\ 1Laboratory of Nanostructures and Nanomaterials, Institute of Physics, AS CR, Na Slovance 2, \\ 18221 Prague 8, Czech Republic \\ ${ }^{2}$ School of Mater. Sci. Eng., University of Science and Technology Beijing, 100083, Beijing, China \\ *lejcekp@fzu.cz
}

(Received : November 11, 2013; Accepted : December 15, 2013)

\begin{abstract}
Segregation volume is an important quantity controlling the pressure effect on grain boundary chemistry. In the present paper, we define molar segregation volume on basis of fundamental thermodynamics and evaluate it for example of phosphorus and sulfur grain boundary segregation in low-alloy ferritic steels including its development during non-equilibrium segregation under tensile and compressive deformations. We also show expected effect of anisotropy on equilibrium segregation under pressure.
\end{abstract}

Dedicated to Prof. Siegfried Hofmann on occasion of his $75^{\text {th }}$ birthday

\section{Introduction}

Grain boundary segregation is a phenomenon affecting many materials properties e.g. cohesion and recrystallization [1]. For its theoretical representation, mainly the Langmuir-McLean segregation isotherm has been used as it operates with thermodynamic quantities like Gibbs energy, enthalpy and entropy $[1,2]$. Unfortunately, no sufficient information has been published about segregation volume, $\Delta V_{I}$, although it controls the grain boundary segregation under pressure or deformation [1]. Lojkowski et al. [3,4] mentioning this quantity in connection to the grain boundary diffusion and wetting under pressure, correctly define $\Delta V_{I}$ as the difference of the crystal volume with the solute segregated at the grain boundary and that without segregation,

$$
\Delta V_{I}=\alpha\left(V_{I}^{G B}-V_{M}^{G B}+V_{M}-V_{I}+V_{r}\right),
$$

where $V_{j}^{\xi}$ is the molar volume of component $j(j=I$, $M$ ) at state $\xi$ ( $\xi$ grain boundary (GB) or bulk), $V_{r}$ is the contribution of changed grain boundary structure due to segregation, and $\alpha$ is the relaxation factor $[3,4]$. Zhang and Ren [5] define $\Delta V_{I}$ as the difference of partial molar volumes of solute $I$ at the grain boundary and in bulk that is not completely correct as they omit the effect of the host component, $M$.

Here, we define the segregation volume, evaluate it for real systems and analyze various effects on this quantity.

\section{Segregation Volume: Definition}

The Langmuir-McLean segregation isotherm relates the grain boundary concentration of solute $I, X_{I}^{G B}$, to its bulk concentration, $X_{I}^{B}$, [1]

$$
\frac{X_{I}^{G B}}{X^{0}-\sum_{j=1}^{M-1} X_{j}^{G B}}=\frac{X_{I}^{B}}{1-\sum_{j=1}^{M-1} X_{j}^{B}} \exp \left(-\frac{\Delta G_{I}}{R T}\right)
$$

where $\Delta G_{I}$ is the molar Gibbs energy of segregation of $I, X^{0}$ is the grain boundary saturation. $\Delta G_{I}$ is principally composed of two terms, the standard molar Gibbs energy of segregation [1,2], $\Delta G_{I}^{0}$, representing the segregation in an ideal system, and the partial molar excess Gibbs energy of segregation, $\Delta \bar{G}_{I}^{E}$, controlled by the difference between the real and ideal behavior, $\Delta G_{I}=\Delta G_{I}^{0}+\Delta \bar{G}_{I}^{E} \cdot \Delta G_{I}^{0}$ is combination of the standard chemical potentials, $\mu_{j}^{0, \zeta}$,

$$
\Delta G_{I}^{0}=\left(\mu_{I(M)}^{0, G B}-\mu_{I(M)}^{0, B}\right)-\left(\mu_{M}^{0, G B}-\mu_{M}^{0, B}\right)
$$




\section{$P$. Lejček et al. Excess Volume in Grain Boundary Segregation}

The standard state is chosen to be pure substance $(M, I)$ at temperature, normal pressure and structure of the host $M$, both in the bulk and at the grain boundary. $\Delta \bar{G}_{I}^{E} \quad$ is defined as

$$
\Delta \bar{G}_{I}^{E}=R T \ln \frac{\gamma_{I}^{G B} \gamma_{M}^{B}}{\gamma_{I}^{B} \gamma_{M}^{G B}}
$$

where $\gamma_{i}^{\zeta}$ are the activity coefficients.

Application of fundamental thermodynamic relationship to the grain boundary segregation provides us with

$$
d \Delta G_{I}=-\Delta S_{I} d T+\Delta V_{I} d P+\sum_{j, \xi} \mu_{j}^{\xi} d X_{i}^{\xi}+\sigma d A
$$

where $\sigma$ is the grain boundary energy, $A$ is the grain boundary molar area, $\Delta S_{I}$ is the molar segregation entropy involving all contributions except the configuration one [1,2]. According to Eq. (5) the molar segregation volume, $\Delta V_{\mathrm{I}}$, is defined as

$$
\Delta V_{I}=\left(\frac{\partial \Delta G_{I}}{\partial P}\right)_{T, A, X_{i}^{\xi}}
$$

$\Delta V_{I}$ is constructed in the same way as other segregation quantities,

$$
\Delta V_{I}=\left(V_{I}^{G B}-V_{I}^{B}\right)-\left(V_{M}^{G B}-V_{M}^{B}\right)
$$

Equation (7) is very similar to Eq. (1) in [3], if $\alpha$ and $V_{r}$ are inherently incorporated in the terms of Eq. (7).

Similarly to $\Delta G_{I}, \Delta V_{I}$ consists of two parts, the standard molar segregation volume, $\Delta V_{I}^{0}$, and the partial molar excess segregation volume, $\Delta \bar{V}_{I}^{E}$, $\Delta V_{I}=\Delta V_{I}^{0}+\Delta \bar{V}_{I}^{E}$. Accepting the chosen standard state,

$$
\Delta V_{I}^{0}=\left(V_{I(M)}^{0, G B}-V_{I(M)}^{0, B}\right)-\left(V_{M}^{0, G B}-V_{M}^{0, B}\right) \equiv 0
$$

as $V_{I(M)}^{0, G B} \equiv V_{M}^{0, G B}$ and $V_{I(M)}^{0, B} \equiv V_{M}^{0, B} \quad$ Therefore, all contributions to $\Delta V_{I}$ originate from the real behavior of the system, i.e. $\Delta V_{I}=\Delta \bar{V}_{I}^{E}$,

$$
\Delta V_{I}=\Delta \bar{V}_{I}^{E}=\left(\bar{V}_{I}^{G B}-\bar{V}_{I}^{B}\right)-\left(\bar{V}_{M}^{G B}-\bar{V}_{M}^{B}\right)
$$

where $\bar{V}_{j}^{\xi}$ are the partial molar volumes of components $j$ at states $\xi$. The pressure effect on grain boundary segregation can be expressed as

$$
\begin{aligned}
& \frac{X_{I}^{G B}(P)}{X^{0}-\sum_{j=1}^{M-1} X_{j}^{G B}(P)}= \\
& \frac{X_{I}^{G B}\left(P=P_{0}\right)}{X^{0}-\sum_{j=1}^{M-1} X_{j}^{G B}\left(P=P_{0}\right)} \exp \left[-\frac{\left(P-P_{0}\right) \Delta \bar{V}_{I}^{E}}{R T}\right]
\end{aligned}
$$

which is principally similar to Eq. (4) in [5].

\section{Segregation Volume: Evaluation}

The relationship between $\Delta \bar{V}_{I}^{E}$ and pressure dependence of $X_{I}^{G B}$ for binary $M-I$ system is obtained from Eqs. (2) and (6) as

$$
\Delta \bar{V}_{I}^{E}=-\frac{R T X^{0}}{X_{I}^{G B}\left(X^{0}-X_{I}^{G B}\right)} \frac{d X_{I}^{G B}}{d P}
$$

Equation (10) may be simplified by $X^{0}=1$ which is often used in quantification of grain boundary segregation [1]. According to Eq. (10), $\Delta \bar{V}_{I}^{E}$ can be obtained from experimental data on pressure/stress dependence of $X_{I}^{G B}$. For example, Shinoda and Nakamura [6] studied the effect of applied elastic stress of $30 \mathrm{MPa}$ on $\mathrm{P}$ grain boundary segregation at $773 \mathrm{~K}$ in a 0.05 wt\% P-doped low-alloy steel by AES. From the Auger peak-to-peak ratios [6] $X_{P}{ }^{G B}$ was determined according to $[7,8]$ as 30.3 at\% under normal pressure and 30.8 at.\% in stressed sample after equilibration supposing simplification of the alloy to binary Fe-P system (Fig. 1). According to Eq. (10), $\Delta \bar{V}_{P}^{E} \approx-5.1$ $\mathrm{ml} / \mathrm{mol}$. On the other hand, no change of $X_{P}{ }^{G B}$ was

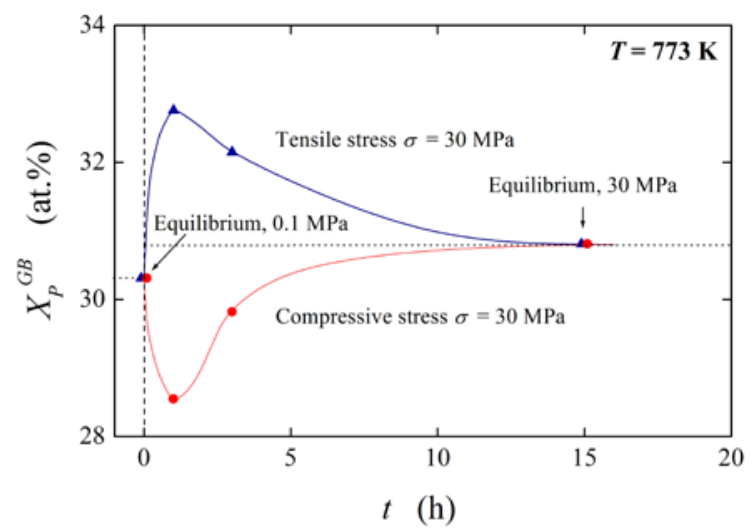

Fig. 1 Time dependence of $X_{P}{ }^{G B}$ in a $0.05 \mathrm{wt} \%$ P-doped low alloy steel at $773 \mathrm{~K}$ under tensile $(\boldsymbol{\Delta})$ or compressive (O) stress of $30 \mathrm{MPa}$ (cf. [6]). 
observed in 2.25Cr1Mo steel [9] under the stress of 40 MPa at $793 \mathrm{~K}$ which implies ideal behavior, $\Delta \bar{V}_{P}^{E}=0$. Surprisingly, the same conclusion results for this system loaded by 100, 200 and $350 \mathrm{MPa}$ which all cause plastic deformation of the material. An increase of $X_{S}^{G B}$ by 0.3 at.\% was observed by Misra [10] in S-doped low-alloy steel stressed plastically at $883 \mathrm{~K}$ by $343 \mathrm{MPa}$, providing the value $\Delta \bar{V}_{S}^{E} \approx-5.5 \mathrm{ml} / \mathrm{mol}$.

The value of $\Delta \bar{V}_{I}^{E}$ can also be estimated in other ways. First, for Eq. (7), $V_{\mathrm{Fe}}{ }^{B}=7.1 \times 10^{-6} \mathrm{~m}^{3} / \mathrm{mol}$ if pure Fe substitutes the $2.6 \mathrm{NiCrMoV}$ steel. As the average boundary density is of about $50 \%$ of the bulk density [11], $V_{F e}^{G B}=1.42 \times 10^{-5} \mathrm{~m}^{3} / \mathrm{mol}$. Based on the density of FeS (4.82 g/ $\mathrm{cm}^{3}$ [12]), $V_{S}^{G B}=1.76 \times 10^{-5} \mathrm{~m}^{3} / \mathrm{mol}$ if $\mathrm{GB}$ is regarded as a $\mathrm{Fe}-48 \mathrm{at} \% \mathrm{~S}$ since the atomic fraction of $\mathrm{S}$ in $2.6 \mathrm{NiCrMoV}$ is $48 \mathrm{at} \%$ after stress-aging for $20 \mathrm{~h}$ [13]. Then $V_{S}^{B}=0.002 \times V_{F e}^{B}=1.4 \times 10^{-9}$ $\mathrm{m}^{3} / \mathrm{mol}$, and $\Delta \bar{V}_{S}^{E}=1.0 \times 10^{-5} \mathrm{~m}^{3} / \mathrm{mol}$. On the other hand, the values of $\Delta \bar{V}_{S}^{E}=-8.1 \times 10^{-6} \mathrm{~m}^{3} / \mathrm{mol}, \Delta \bar{V}_{P}^{E}$ $=-3.0 \times 10^{-6} \mathrm{~m}^{3} / \mathrm{mol}$ and $\Delta \bar{V}_{S e}^{E}=-3.0 \times 10^{-6} \mathrm{~m}^{3} / \mathrm{mol}$ were calculated using the density functional theory technique for segregation of S, P and Se, respectively, at $\{012\}$ grain boundary in $\mathrm{Ni}[14]$. The values of $\Delta \bar{V}_{I}^{E}$ of the order of $10^{-6}$ to $10^{-5} \mathrm{~m}^{3} / \mathrm{mol}$ are low. It suggests that the pressure dependence of $\Delta G_{I}$ and therefore, of $X_{I}^{G B}$ is weak (cf. Eq. (9)). Even the value $\Delta \bar{V}_{I}{ }^{E} \approx-1 \times 10^{-5} \mathrm{~m}^{3} / \mathrm{mol}$ under pressure/stress of 100 MPa causes reduction of $\Delta G_{I}$ by $-1 \mathrm{~kJ} / \mathrm{mol}$, representing a change of $X_{I}^{G B}$ by units of percents. This explains why $X_{I}^{G B}$ measured under pressure differs only slightly from that detected under normal pressure $[6,15,16]$.

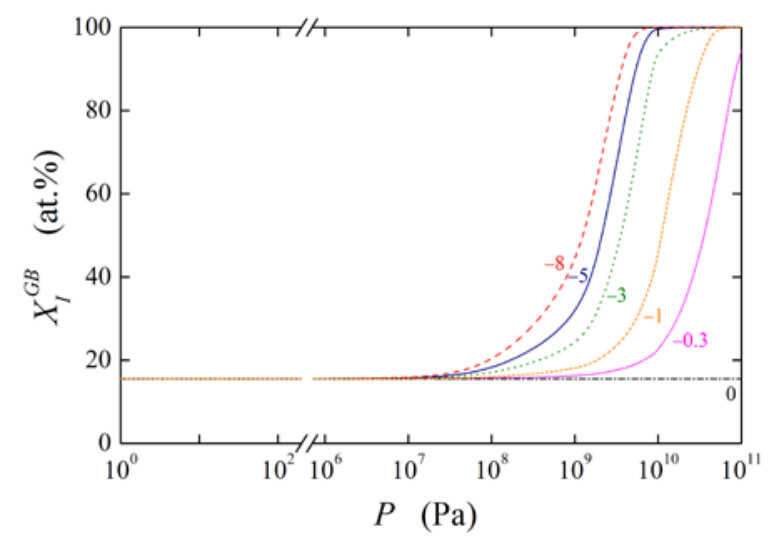

Fig. 2 Dependence of $X_{I}^{G B}$ in a model $M-I(0.01$ at\%) alloy at $800 \mathrm{~K}$ under hydrostatic pressure for various values of $\Delta \bar{V}_{I}^{E}$ (in $\mathrm{ml} / \mathrm{mol}$ ) as indicated.
The effect of $\Delta \bar{V}_{I}^{E}$ on $X_{I}^{G B}$ can be demonstrated on a model example. Supposing the values of the standard enthalpy, entropy of segregation of $I$, and bulk concentration to be $\Delta H_{I}^{0}=-30 \mathrm{~kJ} / \mathrm{mol}, \Delta S_{I}^{0}=+25$ $\mathrm{J} /$ (mol.K) (representing e.g. $\mathrm{P}$ segregation in b.c.c. Fe [1]), and $X_{I}=0.0001$ (i.e. 0.01 at.\%), respectively, $X_{I}^{G B}(0)=0.1554$ (i.e. 15.54 at.\%) under normal pressure at $T=800 \mathrm{~K}$, accepting $X^{0}=1$ in Eq. (9). The pressure dependence of calculated grain boundary concentration is plotted in Fig. 2 for the values of $\Delta \bar{V}_{I}^{E}$ ranging from $-8 \mathrm{ml} / \mathrm{mol}$ to 0 . Figure 2 clearly documents that the changes of $X_{I}^{G B}$ appear when the pressure is higher than $10 \mathrm{MPa}$ : at much higher pressures the increase of $X_{I}^{G B}$ becomes steep. It confirms that the effect of elastic deformation on grain boundary segregation is small but shows clearly that $X_{I}^{G B}$ significantly changes with increasing pressure beyond the elastic limit. During high hydrostatic pressure this effect is rather large: In this case, $X_{I}^{G B}$ should reach the values up to 1 (i.e. 100 at.\%, cf. Fig. 2).

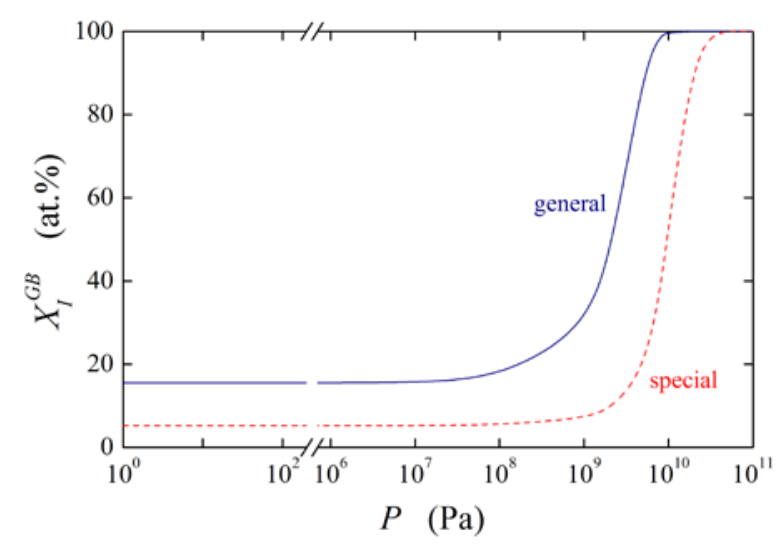

Fig. 3 Dependence of $X_{I}^{G B}$ at special (red, dashed line) and general (blue, full line) grain boundaries in a model $M$ $I(0.01$ at\%) alloy under hydrostatic pressure at $800 \mathrm{~K}$. See text for details.

Similarly to other thermodynamic quantities of segregation [1] we may suppose existence of anisotropy of $\Delta \bar{V}_{I}^{E}$. We may rationally expect that brittle fracture of polycrystals, which has been used for determination of $X_{I}^{G B}$ by means of AES, opens predominantly general boundaries with high tendency to embrittlement. Therefore, we may ascribe the above mentioned value $\Delta \bar{V}_{P}^{E}=-5.1 \times 10^{-6} \mathrm{~m}^{3} / \mathrm{mol}$, determined for $\mathrm{P}$ grain boundary segregation in a $0.05 \mathrm{wt} \%$ P-doped low alloy steel at $773 \mathrm{~K}$ under tensile or 
compressive stress of $30 \mathrm{MPa}$ [6] to general grain boundaries. As its value is negative, the values of $\Delta \bar{V}_{P}^{E}$ for special grain boundaries must be higher (i.e. of lower absolute values). In our model calculation we assumed the values of the thermodynamic parameters of segregation of $I$ to be $\Delta H_{I}^{0}=-30 \mathrm{~kJ} / \mathrm{mol}, \Delta S_{I}^{0}=$ $+25 \mathrm{~J} /(\mathrm{mol} . \mathrm{K})$ and $\Delta \bar{V}_{P}^{E}=-5 \times 10^{-6} \mathrm{~m}^{3} / \mathrm{mol}$ for general grain boundary, and $\Delta H_{I}^{0}=-10 \mathrm{~kJ} / \mathrm{mol}, \Delta S_{I}^{0}=$ $+40 \mathrm{~J} /(\mathrm{mol} . \mathrm{K})$ and $\Delta \bar{V}_{P}^{E}=-2 \times 10^{-6} \mathrm{~m}^{3} / \mathrm{mol}$ for special grain boundary. For $X_{I}=0.0001$, the pressure dependence of $X_{I}^{G B}$ for both types of the grain boundaries at $800 \mathrm{~K}$ is shown in Fig. 3. As expected, the segregation is lower at the special boundary compared to the general one. The concentration change at the special grain boundary requires higher pressure. In both cases, however, complete saturation of the grain boundary is reached under ultimate pressures.

\section{Conclusions}

For the first time, the segregation volume, $\Delta V_{I}$, was thermodynamically defined in this paper. It is shown that the standard molar segregation volume, $\Delta V_{I}^{0} \equiv 0$ at any temperature while the other component of $\Delta V_{I}$, the excess molar volume of segregation $\Delta \bar{V}_{I}^{E} \neq 0$ in real systems. It implies that the grain boundary concentration, $X_{I}^{G B}$, in real systems is affected by pressure. A fundamental relationship between $\Delta \bar{V}_{I}{ }^{E}$ and the pressure change of $X_{I}^{G B}$ was derived. The values of $\Delta \bar{V}_{I}^{E}$ were determined for example of $\mathrm{P}$ and $\mathrm{S}$ grain boundary segregation under stress in bcc-Fe based systems. It was shown that $\Delta \bar{V}_{I}^{E}$ plays an important role in this kind of studies. The effect of anisotropy of $\Delta \bar{V}_{I}^{E}$ is also proposed.

\section{Acknowledgements}

This work was supported by the Czech Science Foundation (P108/12/0144), the National Natural Science Foundation of China (51001011) and the Fundamental Research Funds for the Central Universities (FRF-TP-12-042A).

\section{References}

[1] P. Lejček, Grain Boundary Segregation in Metals, Springer Verlag, Berlin (2010).

[2] P. Lejček and S. Hofmann, Crit. Rev. Sol. State Mater. Sci. 33, 133 (2008).

[3] W. Lojkowski, Def. Diff. Forum 129-130, 269 (1996).

[4] W. Lojkowski, E. Rabkin, B. Straumal and W. Gust, Def. Diff. Forum 143-147, 1407 (1997).

[5] T.-Y. Zhang and H. Ren, Acta Mater. 61, 477 (2013).

[6] T. Shinoda and T. Nakamura, Acta Metall. 29, 1631 (1981).

[7] P. Lejček, Surface Sci. 202, 493 (1988).

[8] P. Lejček, Anal. Chim. Acta 297, 165 (1994).

[9] S.-H. Song, J. Wu, L.-Q. Weng and S.-J. Liu, Mater. Lett. 64, 849 (2010).

[10] R.D.K. Misra, Acta Mater. 44, 885 (1996).

[11] E. Jorra, H. Franz, J. Peisl, W. Petry, G. Wallner, R. Birringer, H. Gleiter, T. Haubold, Phil Mag B, 60, 159 (1989).

[12] J.G. Speight, Lange's Handbook of Chemistry. McGraw-Hill, (2005), Table 4-1.

[13] T.D. Xu, Philos Mag, 87; 1581 (2007),

[14] M. Šob, unpublished results.

[15] T.D. Xu, J. Mater. Sci. 35, 5621 (2000).

[16] S.H. Song, J. Wu, D.Y. Wang, L.Q. Weng and L. Zheng, Mater. Sci. Eng. A 430, 320 (2006). 